\title{
Elektrische Energieversorgung - Wunsch und Wirklichkeit
}

W. Gawlik

Online publiziert am 20. November 2017

C Springer-Verlag GmbH Austria, ein Teil von Springer Nature 2017

Das energiepolitische Zieldreieck umfasst die Aspekte „Wirtschaftlichkeit, Versorgungssicherheit und Umweltverträglichkeit", die zueinander in Konkurrenz stehen. Es ist Aufgabe der Politik, für einen gesellschaftlichen Konsens zu sorgen, der die einander teilweise widersprechenden Aspekte möglichst zusammenführt.

Die aktuellen und zukünftigen Veränderungen im und Herausforderungen an das elektrische Energiesystem machen es notwendig, diesen Konsens laufend neu auszutarieren und dabei auf die Zwän- ge und Chancen, die sich durch technische und gesellschaftliche Entwicklungen ergeben, zu reagieren.

Der Vortrag nimmt eine Bestandsaufnahme vor, zeigt einige historische und gegenwärtige (Fehl-)Entwicklungen auf und soll auch in einem Rückblick auf die Themen und Vorträge der Tagung diese in den Kontext einordnen. 